\title{
High Time Resolution Astronomical Polarimetry with GASP
}

\author{
Eoin G.P. O'Connor ${ }^{1}$, Andrew Shearer ${ }^{1}$, Christian Gouiffes ${ }^{2}$ and \\ Philippe Laurent ${ }^{2}$ \\ ${ }^{1}$ Centre for Astronomy, School of Physics, \\ National University of Ireland Galway, Galway, Ireland \\ email: eoin1. oconnor@gmail.com \\ ${ }^{2}$ CEA/DSM/IRFU/Service d'Astrophysique, C.E.A. Saclay, France
}

\begin{abstract}
The Galway Astronomical Stokes Polarimeter (GASP) is a high time resolution, fullStokes imaging polarimeter which utilises a Fresnel rhomb prism as a beam-splitter and quarter wave retarder. The ability to perform optical photometry and polarimetry at high frame rates enables more detailed studies of a multitude of compact objects including; optical pulsars, magnetars and Active Galactic Nuclei (AGN). We present a brief discussion of the instruments' current capabilities and hardware design. GASP is currently configured with two Electron Multiplying Charge-Coupled Devices (EMCCDs), which offer sub-millisecond frame readout speeds over a defined region of interest on the sensor. We will report results from an observational campaign at the William Herschel Telescope (WHT) in December, 2015. During this campaign GASP was used to study the Crab Pulsar, V404 Cygni and polarisation standards. As a subset of our analysis we have identified a significant contribution from the Interstellar Medium (ISM) and the interaction of a varying polarised source with the ISM, the subsequent conversion of linearly polarised light to circular, and its dependence on the angle of the source emission electric field orientation. Further to the presentation of results we will discuss future observational work, which is planned for September 2017 and subsequent improvements to increase the temporal resolution of the detectors.
\end{abstract}

Keywords. instrumentation: polarimeters, techniques: polarimetric, instrumentation: detectors.

\section{Introduction}

The polarisation state of the Crab Pulsar and its surrounding nebula has been studied for almost fifty years. The linear polarisation of the Crab Pulsar was first discovered in 1969 by Warner et al. (1969) and first presented by Wampler et al. (1969). Shown below (Figure 1) is historical data with a specific focus on the mean position angle of the electric field vector measured for the Crab Pulsar and the background effect on this measurement which is primarily due to the background subtraction method.

\section{Observations and Results}

The data for this analysis was obtained at the WHT in December 2015 and analysed using our own data analysis pipeline which involves an instrument-specific calibration routine and gain analysis on the electron multiplication from both cameras used in the GASP instrument. The Galway Astronomical Stokes Polarimeter (GASP), as a 2D-imaging polarimeter, allows for the selection of varied apertures and background subtraction methodologies. We have demonstrated the effect on the measurements of the pulsar's position angle with respect to the aperture selected and the background 
subtraction method employed for both phase-resolved and integrated data. For the first two measurements we have shown how GASP measurements match previous observations when the same aperture extraction and background subtraction methods are applied and we are continuing this work further.

Table 1: Crab pulsar and nebula. Polarimetry since 1969 .

\begin{tabular}{cccl}
\hline $\begin{array}{c}\text { Observation } \\
\text { Dates }\end{array}$ & Position Angle & Aperture (") & Reference \\
\hline 40296 & $107 \pm 6$ & $5.5 " 9.4 "$ & Wampler et al. $(1969)$ \\
40292 & 89 & $6.8 " 8.1 "$ & Cocke et al. $(1969)$ \\
40512 & 89 & $3 "$ & Cocke et al. $(1970)$ \\
40518 & 98 & & Kristian et al. $(1970)$ \\
$40260,40451-2,4051340558$ & 160 & $*$ & Visvanathan $(1971)$ \\
$40918-9,40972,40975$ & 117 & & Cocke et al. $(1973)$ \\
$41650-3$ & 118 & $5 " 7$ & Ferguson et al. $(1974)$ \\
$44261-2$ & 118 & $2 "$ & Jones et al. $(1981)$ \\
46409 & 130 & $2 "$ & Smith et al. $(1988)$ \\
$52966-71$ & 109.5 & $2.35 "$ & Stowikowskat et al. $(2009)$ \\
$53619-718(53668)$ & 105 & $0.25 "$ & Moran et al. $(2013)$ \\
56243 & 85 & $1.6 " *$ & Moran et al. $(2016)$ \\
57359 & 128 & $2.1 "$ & This work \\
\hline
\end{tabular}

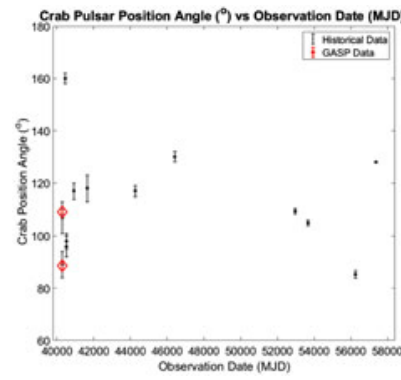

Figure 1: Table 1 data (black point) with GASP data (red diamond)

\section{Acknowledgements}

EOC wishes to acknowledge the support of the Irish Research Council under project funding GOIPG/2014/1266. We also acknowledge the Science Federation of Ireland (SFI) for funding under the SFI Strategic Opportunity Fund for Research Infrastructure and Andor Technology Ltd. for continued technical support. This work was made possible in part through support of the Ulysses Ireland-France collaborative funding research programme. The author also wished to acknowledge funding from RadioNet. RadioNet has received funding from the European Union's Horizon 2020 research and innovation programme under grant agreement No. 730562.

\section{References}

Collins, P., Kyne, G., \& Lara, D., et al. 2013, Experimental Astronomy, 36, 479

Cocke, W. J., Disney, M. J., \& Gehrels, T.1969, Nature, 223, 576

Cocke, W. J., Disney, M. J., \& Muncaster, G. W. 1970, Nature, 227, 1327

Cocke, W. J., Ferguson, D. C., \& Muncaster, G. W. 1973, ApJ, 183, 987

Ferguson, D. C., Cocke, W. J., \& Gehrels, T. 1974, ApJ, 190, 375

Gouiffes, C., Laurent, P., Shearer, A., O'Connor, E., \& Moran, P. 2016, Proceedings of the 11th INTEGRAL Conference Gamma-Ray Astrophysics in Multi-Wavelength Perspective.10-14 October 2016 Amsterdam, The Netherlands (INTEGRAL2016)

Jones, D. H. P., Smith, F. G., \& Wallace, P. T. 1981, MNRAS, 196, 943

Kristian, J., Visvanathan, N., Westphal, J. A., \& Snellen, G. H. 1970, ApJ, 162, 475

Kyne, G., Lara, D., Hallinan, G., Redfern, M., \& Shearer, A. 2016, Experimental Astronomy, 41,43

Moran, P., Shearer, A., Mignani, R. P., et al. 2013, MNRAS, 433, 2564

Moran, P., Kyne, G., \& Gouiffès, C., et al. 2016, MNRAS, 456, 2974

Słowikowska, A., Kanbach, G., Kramer, M., \& Stefanescu, A. 2009, MNRAS, 397, 103

Smith, F. G., Jones, D. H. P.., Dick, J. S. B., \& Pike, C. D. 1988, MNRAS, 233, 305

Wampler, E. J., Scargle, J. D., \& Miller, J. S. 1969, apjl, 157, L1

Warner, B., Nather, R. E., \& Macfarlane, M. 1969, Nature, 222, 233

Visvanathan, N. 1971, The Crab Nebula, 46, 152 